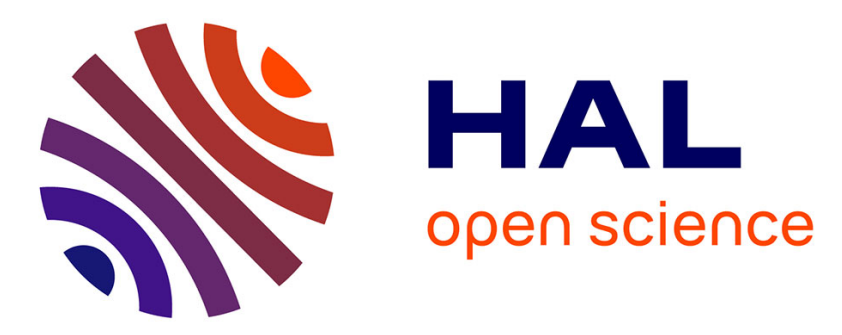

\title{
Torsional Vibrations of Fluid-Filled Multilayered Transversely Isotropic Finite Circular Cylinder
}

\author{
Wafik Abassi, Adil El Baroudi, Fulgence Razafimahéry
}

\section{To cite this version:}

Wafik Abassi, Adil El Baroudi, Fulgence Razafimahéry. Torsional Vibrations of Fluid-Filled Multilayered Transversely Isotropic Finite Circular Cylinder. International Journal of Applied Mechanics, 2016, 8 (3), pp.1650032-1 - 1650032-14. 10.1142/S1758825116500320 . hal-01340514

\section{HAL Id: hal-01340514 \\ https://hal.science/hal-01340514}

Submitted on 4 Feb 2020

HAL is a multi-disciplinary open access archive for the deposit and dissemination of scientific research documents, whether they are published or not. The documents may come from teaching and research institutions in France or abroad, or from public or private research centers.
L'archive ouverte pluridisciplinaire $\mathbf{H A L}$, est destinée au dépôt et à la diffusion de documents scientifiques de niveau recherche, publiés ou non, émanant des établissements d'enseignement et de recherche français ou étrangers, des laboratoires publics ou privés. 


\title{
Torsional Vibrations of Fluid-Filled Multilayered Transversely Isotropic Finite Circular Cylinder
}

\author{
Wafik Abassi* and Adil El Baroudi ${ }^{\dagger}$ \\ Arts et Métiers ParisTech, 2 boulevard du Ronceray \\ 49035 Angers, France \\ *wafik.abassi@ensam.eu \\ †adil.elbaroudi@ensam.eu \\ Fulgence Razafimahery \\ IRMAR, Université de Rennes 1, Campus de Beaulieu \\ 35042 Rennes Cedex, France \\ fulgence.razafimahery@univ-rennes1.fr
}

\begin{abstract}
An analytical and numerical study for the torsional vibrations of viscous fluid-filled three-layer transversely isotropic cylinder is presented in this paper. The equations of motion of solid and fluid are respectively formulated using the constitutive equations of a transversely isotropic cylinder and the constitutive equations of a viscous fluid. The analytical solution of the frequency equation is obtained using the boundary conditions at the free surface of the solid layer and the boundary conditions at the fluid-solid interface. The frequency equation is deduced and analytically solved using the symbolic Software Mathematica. The finite element method using Comsol Multiphysics Software results are compared with present method for validation and an acceptable match between them were obtained. It is shown that the results from the proposed method are in good agreement with numerical solutions. The influence of fluid dynamic viscosity is thoroughly analyzed and the effect of the isotropic properties on the natural frequencies is also investigated.
\end{abstract}

Keywords: Fluid-structure interactions; transversely isotropic cylinders; viscous fluid; finite element method.

\section{Introduction}

A structure in contact with dense fluid (submerged and/or subjected to liquid) has the vibrational characteristics affected by the adjacent fluid. Especially in the torsional vibration of a circular structure in contact with a viscous fluid, the motion of the solid exerts shear stress to the viscous fluid. Meanwhile the fluid resists the motion of the solid, and in so doing the vibrational characteristics of the structure are influenced. The indepth study of various vibrational characteristics, as a function of material and geometrical parameters is necessary for a wide range of applications, 
from physiology of blood flow through an artery, non-destructive evaluation of oil and gas pipelines, measurement of the fluid viscosity, to the geophysical prospecting in cased holes.

The most general form of harmonic waves in a hollow cylinder of circular crosssection has been analyzed by Gazis [1959a, 1959b]. Mirsky [1965] has considered the most general type of elastic wave propagation in both hollow and solid circular cylinders of transversely isotropic material. Torsional motion has been discussed as a special case. Armenakas [1965] has also studied torsional waves in composite elastic rods.

The case of torsional vibrations of transversely isotropic hollow circular cylinders in vacuum has been considered [Carcione et al., 1994] who were interested in the inspection of material properties effects on the phase velocity of the torsional modes. Kuldlicka [2006] has studied the frequency equations for torsional vibrations. Misra [1983] investigated the propagation of torsional waves in tubular bones. Kaul et al. [1981] presented the free vibration analysis for axially asymmetric torsional waves in an elastic, bimetallic rod with cylindrical core and concentric outer casing.

The case of interaction between the torsional stress waves and the viscous fluid has been considered by Kim et al. [1991, 1993]. The research interested in the inspection of the effect of the adjacent viscous fluid on the propagation speed and attenuation of the torsional wave, and suggested viscosity measurement using torsional wave.

In most studies mentioned above, the frequency equation of the vibration problem is obtained without taking the effect of the fluid into account. The originality of this paper is to investigate the effect of viscous fluid on the modal analysis of three layered fibers placed in an finite transversely isotropic cylinder (see Fig. 1). This paper also discusses the influence of the properties of the multi-layered medium on the natural frequencies. Indeed, the free vibration is studied using the wave propagation method and the frequency equation is obtained. Comparisons of the results by the present method with those obtained by numerical finite element method have been carried out. It is shown that the present approach is simple, correct and also gives reasonably accurate natural frequencies.

\section{Theoretical Model of Transversely Isotropic Layers}

\subsection{Governing equations}

Consider a transversely isotropic circular cylinder of finite length with three layers filled with viscous fluid in the cylindrical coordinate system $(r, \theta, z)$. Note that the $z$-axis is taken along the direction of fibers. The material parameters such as the elastic modulus, Poisson's ratio, and the thickness of a layer may be different in different layers. Figure 1(a) shows the cross-section of a three-layer elastic cylinder filled with viscous fluid. The thickness of every layer is determined by radius $a$, $a_{1}, a_{2}$ and $a_{3}$ where $a$ and $a_{3}$ are the radius of the inner and outer surfaces of the 


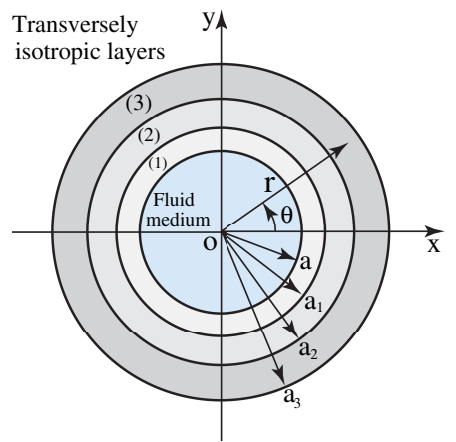

(a) The cross-section of the system

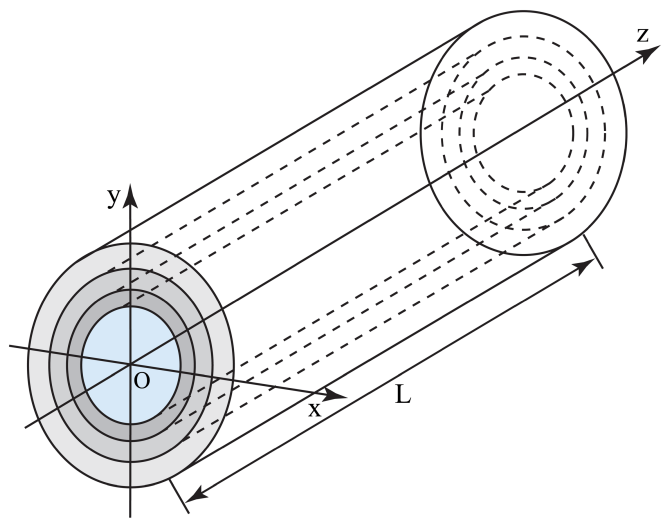

(b) Three-dimensional geometry

Fig. 1. The geometry of the cylinder with 3-layers filled with viscous fluid.

cylinder, respectively. It is assumed that the cylinder is free on the outer surface. The layers are assumed to be perfectly bonded. The system displacements and stresses are defined by the cylindrical coordinates $r, \theta$ and $z$. The length of the cylinder is $L(0 \leq z \leq L)$. The values related to the inner, middle and external cylinders will be denoted by the upper indices (1), (2) and (3), respectively.

In the case of a torsional vibration problem, all the quantities are independent of $\theta$. The nonzero component of displacement is $u_{\theta}$. In the absence of body forces, the equation describing the motion of torsional vibrations is governed by

$$
\frac{\partial \sigma_{r \theta}^{(i)}}{\partial r}+\frac{2 \sigma_{r \theta}^{(i)}}{r}+\frac{\partial \sigma_{z \theta}^{(i)}}{\partial z}=\rho_{i} \frac{\partial^{2} u_{\theta}^{(i)}}{\partial t^{2}} \quad(i=1,2,3)
$$

where $\rho_{i}$ the mass density, $\sigma_{\theta z}^{(i)}, \sigma_{r \theta}^{(i)}$, the stress components, $u_{\theta}^{(i)}$ the displacement components along circumferential directions, and $t$ the time.

Consider a three homogeneous and transversely isotropic cylinders with the two end surfaces parallel to the plane of isotropy. For the cylindrical coordinate system $(r, \theta, z)$ shown in Fig. 1, the generalized Hooke's law for transversely isotropic solids can be written as

$$
\sigma_{\theta z}^{(i)}=2 C_{44}^{(i)} \varepsilon_{\theta z}^{(i)}, \quad \sigma_{r \theta}^{(i)}=2 C_{66}^{(i)} \varepsilon_{r \theta}^{(i)},
$$

where $\varepsilon_{\theta z}^{(i)}, \varepsilon_{r \theta}^{(i)}$ are the strain components, $C_{44}^{(i)}$ and $C_{66}^{(i)}$ are the elastic coefficients of layer $i$. The strains components related to the displacements are given by geometrical relations

$$
\varepsilon_{\theta z}^{(i)}=\frac{1}{2} \frac{\partial u_{\theta}^{(i)}}{\partial z}, \quad \varepsilon_{r \theta}^{(i)}=\frac{1}{2}\left(\frac{\partial u_{\theta}^{(i)}}{\partial r}-\frac{u_{\theta}^{(i)}}{r}\right) .
$$


Substituting Eq. (2) in Eq. (1) and taking into account Eq. (3), yields the following second order partial differential equation with constant coefficients

$$
\frac{\partial^{2} u_{\theta}^{(i)}}{\partial r^{2}}+\frac{1}{r} \frac{\partial u_{\theta}^{(i)}}{\partial r}-\frac{u_{\theta}^{(i)}}{r^{2}}+\frac{C_{44}^{(i)}}{C_{66}^{(i)}} \frac{\partial^{2} u_{\theta}^{(i)}}{\partial z^{2}}=\frac{\rho_{i}}{C_{66}^{(i)}} \frac{\partial^{2} u_{\theta}^{(i)}}{\partial t^{2}}, \quad(i=1,2,3) .
$$

Now, to yield a solution for the proposed problem, the specific boundary and continuity conditions that have to be satisfied at the interface of three-layer transverse isotropic cylinder filed with viscous fluid are, respectively, written as

- At the solid-fluid interface, the continuity of the stress and velocity of the solid and fluid is given by

$$
\left.\sigma_{r \theta}^{(1)}\right|_{r=a}=\left.\sigma_{r \theta}^{(f)}\right|_{r=a},\left.\quad j \omega u_{\theta}^{(1)}\right|_{r=a}=\left.v_{\theta}\right|_{r=a}
$$

where $v_{\theta}$ is the fluid velocity components along circumferential directions and $\sigma_{r \theta}^{(f)}$ the fluid viscous stress components.

- The outside $(i=3)$ surface of the three-layer cylinder are completely stress free. At the internal interfaces of layers $(i=1)$ and $(i=2)$, and also at $(i=2)$ and $(i=3)$, there is equality of each displacement and of stress

$$
\begin{array}{ll}
\left.\sigma_{r \theta}^{(1)}\right|_{r=a_{1}}=\left.\sigma_{r \theta}^{(2)}\right|_{r=a_{1}}, & \left.u_{\theta}^{(1)}\right|_{r=a_{1}}=\left.u_{\theta}^{(2)}\right|_{r=a_{1}} \\
\left.\sigma_{r \theta}^{(2)}\right|_{r=a_{2}}=\left.\sigma_{r \theta}^{(3)}\right|_{r=a_{2}}, & \left.u_{\theta}^{(2)}\right|_{r=a_{2}}=\left.u_{\theta}^{(3)}\right|_{r=a_{2}} \\
\left.\sigma_{r \theta}^{(3)}\right|_{r=a_{3}}=0 &
\end{array}
$$

We therefore examine the equations of motion for the fluid

$$
\frac{\partial \sigma_{r \theta}^{(f)}}{\partial r}+\frac{2 \sigma_{r \theta}^{(f)}}{r}+\frac{\partial \sigma_{z \theta}^{(f)}}{\partial z}=\rho_{f} \frac{\partial v_{\theta}}{\partial t}
$$

where $\rho_{f}$ is the density of the fluid. Since the value of amplitude of vibration of each transversely isotropic circular cylinder is small compared to its cross-sectional dimensions, it then follows that all nonlinear convective inertial effects in the fluid can be neglected. Moreover, the hydrodynamic loading on the transversely isotropic circular cylinder will be a linear function of its velocity. This implies that the fluid dynamics can be modeled as an unsteady linear Stokes flow. Assuming that the fluid is Newtonian, its constitutive equation is given by

$$
\sigma_{r \theta}^{(f)}=\eta\left(\frac{\partial v_{\theta}}{\partial r}-\frac{v_{\theta}}{r}\right)
$$

where $\eta$ is the dynamic fluid viscosity. Substituting Eq. (8) in Eq. (7), yields the following second order partial differential equation:

$$
\frac{\partial^{2} v_{\theta}}{\partial r^{2}}+\frac{1}{r} \frac{\partial v_{\theta}}{\partial r}-\frac{v_{\theta}}{r^{2}}+\frac{\partial^{2} v_{\theta}}{\partial z^{2}}=\frac{1}{\nu} \frac{\partial v_{\theta}}{\partial t}
$$

in which $\nu=\eta / \rho_{f}$ is the kinematic fluid viscosity.

In this way, investigation of the considered wave dispersion problem is reduced to the study of the eigenvalue problem formulated through the Eqs. (4) and (7) and conditions (5) and (6). 


\subsection{Solution method}

For the investigation of the dynamical problem of three-layer transversely isotropic cylinder filed with viscous fluid, various solution methods, including numerical (FEM and BEM for example) and analytical are used. In the present study, for the solution of the problem under consideration, we use the analytical and numerical method, according to which, first, the exact solution of the problem is found and then the eigenvalue equations are obtained from the corresponding contact and boundary conditions. Finally, the solution of this eigenvalue equation is found numerically using Comsol Multiphysics [Comsol, 2008] Software. Assuming harmonic time variations, and using the classical technique of separation of variables, the displacement and velocity should be of the following form:

$$
\begin{aligned}
u_{\theta}^{(i)}(r, z, t) & =U_{i}(r) \cos (k z) \exp (j \omega t) \\
v_{\theta}(r, z, t) & =V(r) \cos (k z) \exp (j \omega t)
\end{aligned}
$$

in which $k=m \pi / L$ is the wave number in the $z$-direction, $L$ is the length of the cylinder, $\omega$ is the angular frequency and $j=\sqrt{-1}$. Substituting expressions (10) into the Eq. (4) we obtain the following equation for $U_{i}(r)$ and $V(r)$ :

$$
\begin{aligned}
\frac{d^{2} U_{i}(r)}{d r^{2}}+\frac{1}{r} \frac{d U_{i}(r)}{d r}+\left(\beta_{i}^{2}-\frac{1}{r^{2}}\right) U_{i}(r) & =0 \\
\frac{d^{2} V(r)}{d r^{2}}+\frac{1}{r} \frac{d V(r)}{d r}+\left(\delta^{2}-\frac{1}{r^{2}}\right) V(r) & =0
\end{aligned}
$$

In Eq. (11) the radial wave numbers $\beta_{i}$ and $\delta$ can be written in the following form:

$$
\beta_{i}^{2}=k^{2}\left(\frac{c_{p}^{2}}{c_{T_{i}}^{2}}-\frac{C_{44}^{(i)}}{C_{66}^{(i)}}\right), \quad \delta^{2}=-\frac{j \omega}{\nu}-k^{2}
$$

where $c_{p}=\omega / k$ is the phase velocity and $c_{T_{i}}=\sqrt{C_{66}^{(i)} / \rho_{i}}$ is the velocity of shear elastic wave in the multilayered transverse isotropic cylinder. Equation (12) shows that the torsional frequencies are crucially dependent on the ratio of the material constants $C_{44}^{(i)} / C_{66}^{(i)}$. The parameter $k c_{T_{i}} \sqrt{C_{44}^{(i)} / C_{66}^{(i)}}$ defined by Eq. (12) is a resonance frequency parameter when the radial wave number $\beta_{i}$ is taken equal to zero. The frequencies are the cut-off frequencies and can be plotted as functions of the ratio $C_{44}^{(i)} / C_{66}^{(i)}$, for different values of the longitudinal wave number $m$. Equation (11) is the general form of Bessel's differential equations and its general solution is presented as follows:

$$
U_{i}(r)=A_{i} J_{1}\left(\beta_{i} r\right)+B_{i} Y_{1}\left(\beta_{i} r\right), \quad V(r)=C J_{1}(\delta r)
$$

where $J_{1}$ is the Bessel function of the first kind and $Y_{1}$ is the Bessel function of the second kind. $A_{i}, B_{i}$ and $C$ are real unknown coefficients. The solution of the equation of motion given by Eqs. (4) and (9) may be applied directly to each layer of the cylinder. Thus, the complete solution for three-layer cylinder will contain six 
integration constants $A_{i}$ and $B_{i}(i=1,2,3)$. These constants may be determined by requiring the solution to satisfy the boundary and continuity conditions of cylinder Eqs. (5) and (6). The general solution of $u_{\theta}^{(i)}$ depends on whether $c_{p}^{2} / c_{T_{i}}^{2}$ greater than $C_{44}^{(i)} / C_{66}^{(i)}$. However, we do not attempt to consider all the possible scenarios here. Because once the elastic constants are given, the general form for $u_{\theta}^{(i)}$ in different frequency ranges can be identified accordingly. Direct substitution of the expression (13) with (10) into the stress relations (2) and (8), leads to

$$
\begin{aligned}
\sigma_{r \theta}^{(i)} & =C_{66}^{(i)}\left\{A_{i}\left[J_{1}^{\prime}\left(\beta_{i} r\right)-\frac{J_{1}\left(\beta_{i} r\right)}{r}\right]+B_{i}\left[Y_{1}^{\prime}\left(\beta_{i} r\right)-\frac{Y_{1}\left(\beta_{i} r\right)}{r}\right]\right\} \cos (k z) \exp (j \omega t) \\
\sigma_{r \theta}^{(f)} & =\eta C\left[J_{1}^{\prime}(\delta r)-\frac{J_{1}(\delta r)}{r}\right] \cos (k z) \exp (j \omega t)
\end{aligned}
$$

where the prime signifies differentiation with respect to the argument (i.e., $\beta_{i} r$ or $\delta r$ ). Thus, frequency (or wave speed) equation in the the multilayered transversely isotropic circular cylinder will be investigated by the use of Eqs. (5) and (6).

Note that for an isotropic circular cylinder the set of equations (4) simplify and can be expressed as follows [Morse et al., 1946]:

$$
\frac{\partial^{2} u_{\theta}^{(i)}}{\partial r^{2}}+\frac{1}{r} \frac{\partial u_{\theta}^{(i)}}{\partial r}-\frac{u_{\theta}^{(i)}}{r^{2}}+\frac{\partial^{2} u_{\theta}^{(i)}}{\partial z^{2}}=\frac{\rho_{i}}{\mu_{i}} \frac{\partial^{2} u_{\theta}^{(i)}}{\partial t^{2}}
$$

where $\mu_{i}$ is the Lamé constant and Eq. (12) becomes

$$
\beta_{i}^{2}=k^{2}\left(\frac{c_{p}^{2}}{c_{T_{i}}^{2}}-1\right)
$$

where $c_{T_{i}}=\sqrt{\mu_{i} / \rho_{i}}$ is the velocity of shear elastic wave in the isotropic layer.

\subsection{Frequency equation}

In this section, we shall derive the frequency (or wave speed) equation for the multilayered transversely isotropic circular cylinder. Combining the continuity and boundary conditions (5) and (6) with Eqs. (10), (13) and (14) yields for each mode number $m$ the following linear system

$$
M \mathbf{x}=\mathbf{0}, \quad \mathbf{x}^{\mathrm{T}}=\left[C, A_{1}, B_{1}, A_{2}, B_{2}, A_{3}, B_{3}\right]
$$

The matrix $\mathbf{M}$ is defined as follows:

$$
\mathbf{M}=\left[\begin{array}{ccccccc}
-M_{11} & M_{12} & M_{13} & 0 & 0 & 0 & 0 \\
M_{21} & M_{22} & M_{23} & 0 & 0 & 0 & 0 \\
0 & M_{32} & M_{33} & -M_{34} & -M_{35} & 0 & 0 \\
0 & M_{42} & M_{43} & -M_{44} & -M_{45} & 0 & 0 \\
0 & 0 & 0 & M_{54} & M_{55} & -M_{56} & -M_{57} \\
0 & 0 & 0 & M_{64} & M_{65} & -M_{66} & -M_{67} \\
0 & 0 & 0 & 0 & 0 & M_{76} & M_{77}
\end{array}\right]
$$


and whose elements are given in the appendix. For a nontrivial solution, the determinant of the matrix $M$ must be equal to zero. Thus, the frequency equation can be obtained

$$
\left|M_{p q}\right|=0 \quad(p, q=1,2, \ldots, 7)
$$

For given material and geometric properties, the frequency equation (16) is a transcendental relation between the angular frequency $\omega$ and the wave number $k$. For any value of $k$, the frequency equation will yield an infinite number of values of $\omega$, each corresponding to a different mode of wave propagation.

\section{Results and Validation}

We consider the three-layer circular cylinder filled with a viscous fluid shown in Fig. 1 and assume that the radius of the internal circle of the inner cylinder is $a$ and the thickness of the inner, middle and outer cylinders is $h_{1}=a_{1}-a, h_{2}=a_{2}-a_{1}$ and $h_{3}=a_{3}-a_{2}$, respectively. The resulting frequency equations of the three-layer model, one-layer model and coupled model cases of the cylinder is given in Eq. (16) are transcendental in nature with respect to the frequency $\omega$ and wavenumber $\beta$ and $\delta$. To obtain the roots of the frequency equation, the Mathematica Software [Mathematica, 2010] is used. To validate the analytical results, the natural frequencies and mode shapes are also computed using Comsol Multiphysics FEM Simulation Software [Comsol, 2008].

In One-layer model case, we show an example of an equal-material properties structure and thickness equal to $a_{3}-a$. Thus, we consider the geometric and material properties of finite circular cylinder given by Kudlicka [2006] without fluid. In the three-layer model case, we have replaced the middle layer by an isotropic layer. As an example, we consider the geometric and material properties of finite circular cylinder given by Markus et al. [1995] without a viscous fluid. In this example, the three-layer cylinder, has the inner and outer transversely isotropic layers of equal thickness $h_{1}=h_{3}$. The thickness of this middle layer is $h_{2}=h_{1} / 10$. The outer radius to the total thickness ratio $a_{3} / h=2$ and $h=h_{2}+2 h_{1}$. The mechanical properties of the transversely isotropic layer are listed in Table 1 and the following values of the the isotropic layer (middle layer $i=2$ ) were assumed: $\rho_{2}=1050\left[\mathrm{~kg} / \mathrm{m}^{3}\right]$ (density), $\nu_{2}=0.48$ (Poisson ration) and $E_{2}=0.3[\mathrm{GPa}]$ (Young's modulus).

Table 1. The material and geometric properties of the three-layer transversely isotropic cylinder filled with fluid.

\begin{tabular}{lccccc}
\hline Property & $C_{44}^{(i)}[\mathrm{GPa}]$ & $C_{66}^{(i)}[\mathrm{GPa}]$ & $\rho_{i}\left[\mathrm{~kg} / \mathrm{m}^{3}\right]$ & $h_{i} / a$ & $\eta[\mathrm{Pa} \cdot \mathrm{s}]$ \\
\hline Inner layer $(i=1)$ & 5 & 6 & 1576 & $10 / 21$ & \\
Middle layer $(i=2)$ & 5 & 6 & 1576 & $1 / 21$ & \\
Outer layer $(i=3)$ & 5 & 6 & 1576 & $10 / 21$ & \\
Fluid & & & 1000 & 1 & 1 \\
\hline
\end{tabular}


Table 2. First three roots $\omega$ of the frequency equation (16) in the case of one-layer transversely isotropic cylinder filled with fluid for $m=1,2,3$ and $\eta=1[\mathrm{~Pa} \cdot \mathrm{s}]$.

\begin{tabular}{cllc}
\hline $\begin{array}{c}\text { Longitudinal mode } \\
\text { number }(m)\end{array}$ & Present & FEM & $100 \times($ FEM-Present $) /$ Present \\
\hline 1 & 0.0147258 & 0.014727 & 0.008 \\
1 & 0.0492623 & & \\
1 & 0.103543 & & 0.004 \\
2 & 0.0148574 & 0.014858 & \\
2 & 0.0493939 & & 0.007 \\
2 & 0.103675 & & \\
3 & 0.0150768 & 0.015078 & \\
3 & 0.0496132 & & \\
3 & 0.103894 & & \\
\hline
\end{tabular}

Table 3. First three roots $\omega$ of the frequency equation (16) in the case of three-layer transversely isotropic cylinder filled with fluid for $m=1,2,3$ and $\eta=1[\mathrm{~Pa} \cdot \mathrm{s}]$.

\begin{tabular}{cllc}
\hline $\begin{array}{c}\text { Longitudinal mode } \\
\text { number }(m)\end{array}$ & Present & FEM & $100 \times($ FEM-Present $) /$ Present \\
\hline 1 & 0.0147258 & 0.014727 & 0.008 \\
1 & 0.0492623 & & \\
1 & 0.103543 & & 0.004 \\
2 & 0.0148574 & 0.014858 & \\
2 & 0.0493939 & & 0.007 \\
2 & 0.103675 & & \\
3 & 0.0150768 & 0.015078 & \\
3 & 0.0496132 & & \\
3 & 0.103894 & & \\
\hline
\end{tabular}

For the torsional natural frequencies of viscous fluid-filled three-layer transversely isotropic cylinder, the following concluding remarks could be drawn:

- Tables 2 and 3 show the comparison of analytically and numerically obtained natural frequencies. The very good agreement is observed between the results of the present method and those of FEM and the relative difference ((FEMPresent)/Present) is $\leq 0.1 \%$. Tables 2 and 3 also shows that the frequencies are in order with the modal parameter $m$. The frequency of mode $(m=1)$ is lower than that of mode $(m=2)$ for example.

- In the case of one-layer transversely isotropic cylinder without fluid, the results in the Table 4 are identical to those presented in Kudlicka [2006], indicating the validity of our Model.

- This is shown in Table 5, in the case of three-layer model in vacuo, for $m=1,2,3$, where results are given for different values of the middle layer stiffness $E_{2}$, that the natural frequencies are perceptibly altered by change in the middle layer stiffness. However, in the coupled model case, the middle layer stiffness $E_{2}$ does not have any significant influence on the natural frequency and the two models (the three-layer and one-layer model) are hence almost equivalent as shown in Tables 2 and 3 . 
Table 4. First three roots $\beta$ of the frequency equation (16) in the case of one-layer transversely isotropic cylinder for $m=1,2,3$.

\begin{tabular}{cccr}
\hline $\begin{array}{c}\text { Longitudinal mode number } \\
(m)\end{array}$ & $\begin{array}{c}\text { Present } \\
\beta\end{array}$ & $\begin{array}{c}\text { [Kuldlicka, 2006] } \\
\beta\end{array}$ & \multicolumn{1}{c}{ Present } \\
& $\omega$ \\
\hline 1 & 3.4069 & 3.407 & 6657.98 \\
1 & 6.4277 & 6.428 & 12547.28 \\
1 & 9.5228 & 9.523 & 18584.55 \\
2 & 3.4069 & & 6689.26 \\
2 & 6.4277 & & 12563.90 \\
2 & 9.5228 & & 18595.78 \\
3 & 3.4069 & & 6741.07 \\
3 & 6.4277 & & 12591.58 \\
3 & 9.5228 & & 18614.48 \\
\hline
\end{tabular}

Table 5. Influence of the middle layer stiffness $E_{2}[\mathrm{GPa}]$ on the $\omega$ in the case of three-layer transversely isotropic cylinder for $m=1$ in vacuo.

\begin{tabular}{lccrrrrr}
\hline$m$ & $E_{2}=0.1$ & $E_{2}=0.5$ & $E_{2}=1$ & $E_{2}=2$ & $E_{2}=3$ & $E_{2}=4$ & $E_{2}=5$ \\
\hline 1 & 1487.5 & 3039.7 & 3976.3 & 4938.9 & 5441.3 & 5749.5 & 5957.2 \\
1 & 11056.2 & 12640.2 & 12695.2 & 12719.2 & 12726.8 & 12730.6 & 12732.8 \\
1 & 13102.0 & 13668.5 & 14253.7 & 15206.4 & 15918.9 & 16455.6 & 16866.9 \\
2 & 1619.36 & 3106.63 & 4027.8 & 4980.67 & 5479.3 & 5785.5 & 5992.0 \\
2 & 11061.3 & 12655.2 & 12710.5 & 12734.7 & 12742.5 & 12746.3 & 12748.7 \\
2 & 13117.3 & 13683.4 & 14268.1 & 15219.9 & 15931.9 & 16468.2 & 16879.3 \\
3 & 1817.9 & 3214.9 & 4112.2 & 5049.4 & 5542.0 & 5845.0 & 6049.5 \\
3 & 11069.8 & 12680.3 & 12736.0 & 12760.6 & 12768.5 & 12772.6 & 12775.1 \\
3 & 13142.8 & 13708.2 & 14292.1 & 15242.5 & 15953.6 & 16489.2 & 16899.8 \\
\hline
\end{tabular}

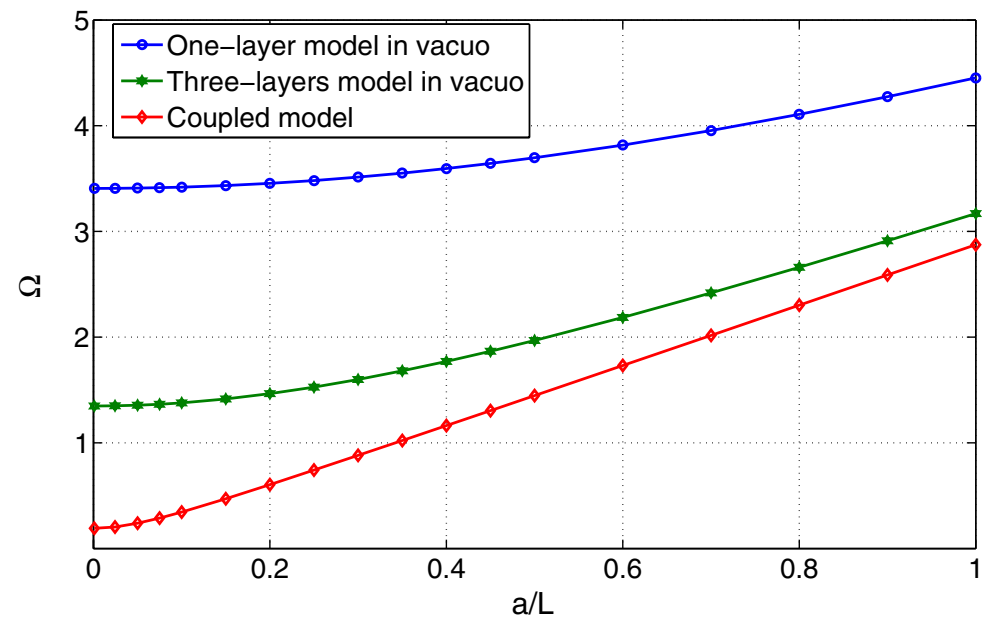

Fig. 2. The frequency curves for different models, as $\Omega=\omega a / c_{T}$ versus $a / L$.

- The frequencies given in Tables 2-5 are restricted to circular cylinders with $L / a=15$. To study the dependence of frequencies on the geometric ratio of the cylinder, we plot the normalized frequency, $\omega a / c_{T}$, against $L / a$ in Fig. 2. The frequency spectrum for the vibration modes is given in Fig. 2 for the three 


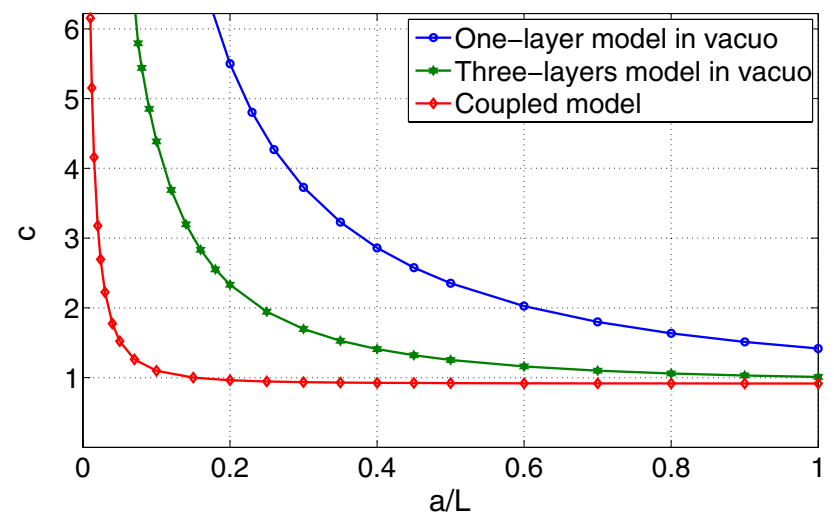

Fig. 3. The phase velocity $c=c_{p} / c_{T}$ dispersion curves in function of $a / L$ for different models.

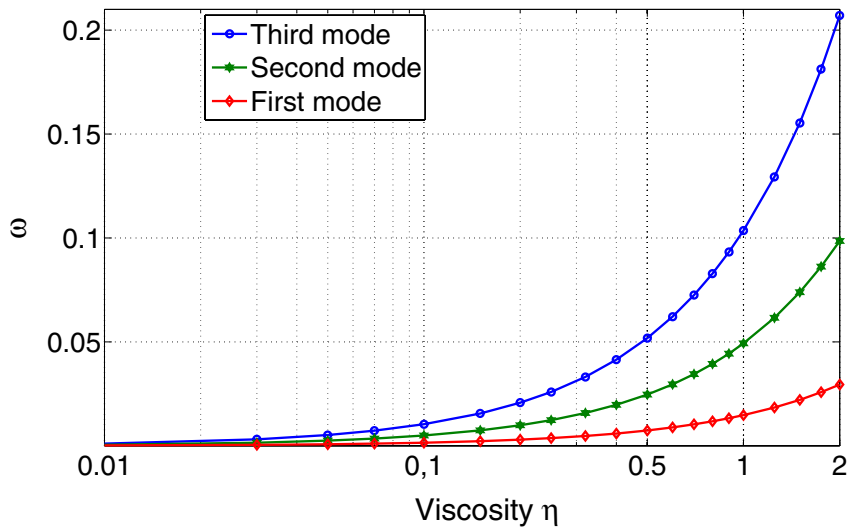

Fig. 4. First curves of the frequency in function of liquid viscosity for various modes.

models considered. Only the first mode (i.e., $m=1$ ) is shown. It is noted that the frequency spectrum for the vibration modes $m=2$ and 3 has similar behavior to that of $m=1$.

- Figure 2 gives the frequency spectrum $\Omega=\omega a / c_{T}$ of a cylinder for, respectively, one-layer model in vacuo, three-layer model in vacuo and the coupled model. $c_{T}$ denotes a reference velocity given by $: \sqrt{C_{66} / \rho}$. The intersection of a mode curve with the frequency axis $(\Omega)$ indicates a cut-off in the sense that it is a propagation limit, i.e., a resonance with infinite wavelength.

- Figure 3 shows the phase velocity $(c)$ dispersion curve of three models adopted in this study with $L / a=15$, where $m=1$. The dependence of phase velocities $c$ upon the ratio $a / L$ and nature of model adopted is clearly illustrated. This behavior is also found for $m=2$ and $m=3$.

- Figure 4 illustrates the influence of the dynamic viscosity of fluid on the angular frequencies with $m=1$. As expected, Fig. 4 indicates that the angular frequencies 


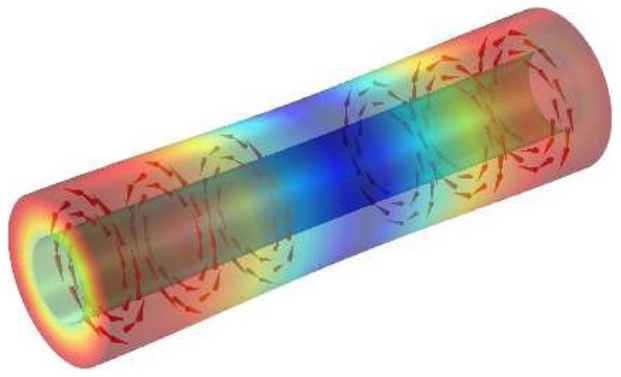

(a) One-layer model in vacuo

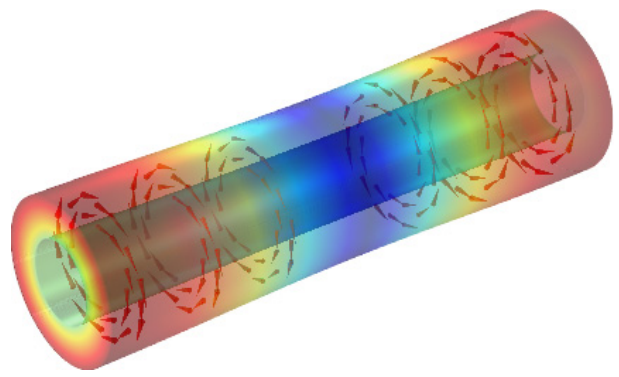

(b) Three-layer model in vacuo

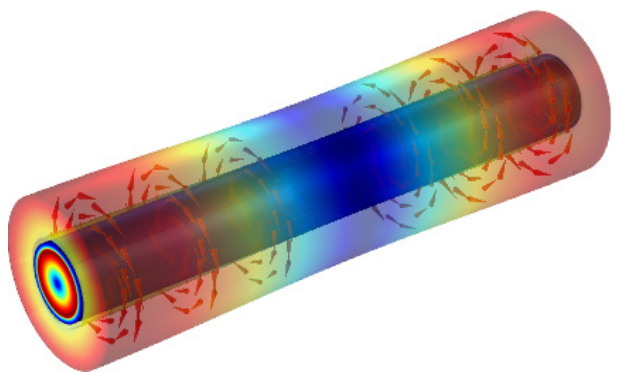

(c) Coupled model

Fig. 5. Modal shapes of the three models for $m=1$ : the colors pertain to the displacement field.

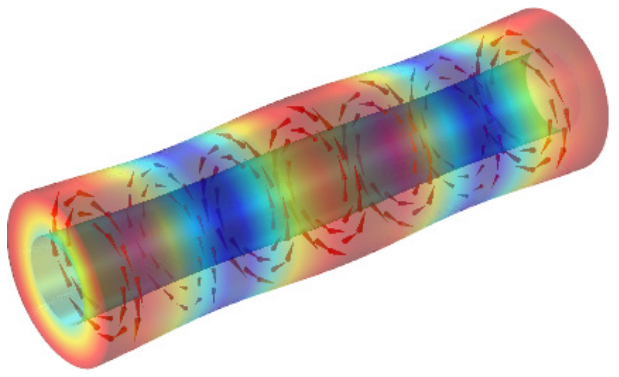

(a) One-layer model in vacuo

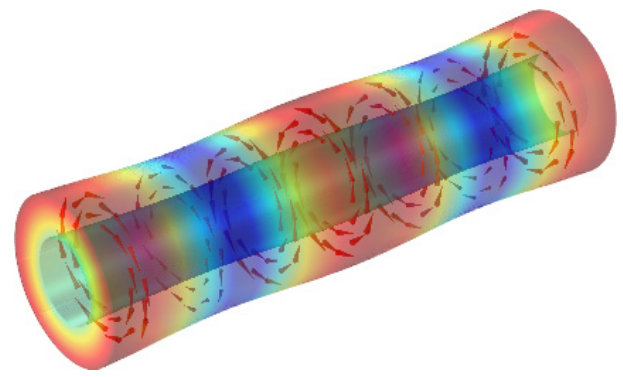

(b) Three-layer model in vacuo

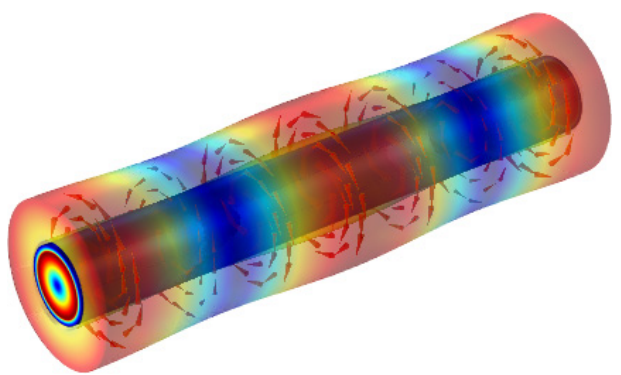

(c) Coupled model

Fig. 6. Modal shapes of the three models for $m=2$ : the colors pertain to the displacement field. 


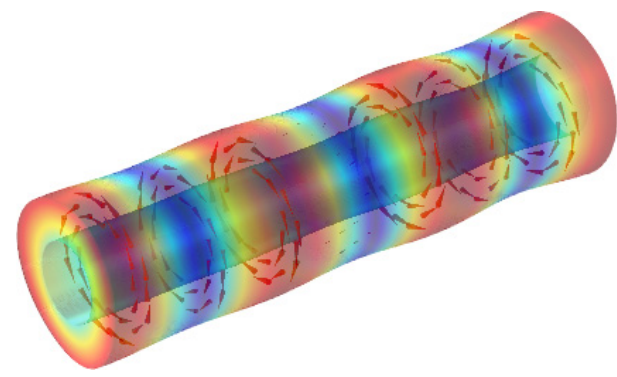

(a) One-layer model in vacuo

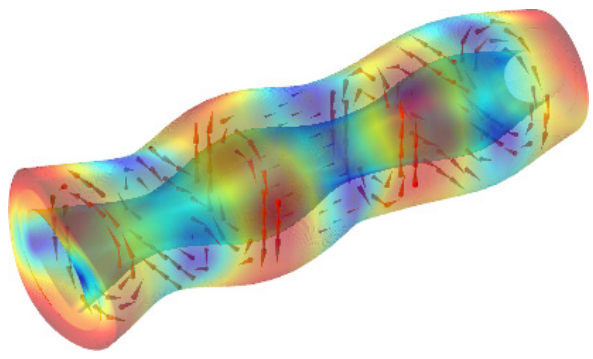

(b) Three-layer model in vacuo

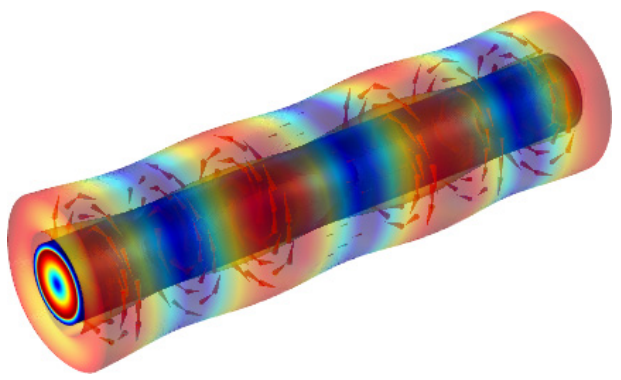

(c) Coupled model

Fig. 7. Modal shapes of the three models for $m=3$ : the colors pertain to the displacement field.

increase with the increase of the dynamic viscosity of fluid. This behavior is also found for $m=2$ and $m=3$.

○ In Figs. 5-7 we give some mode shapes of the three models adopted in this study. These mode shapes are identical to those obtained in El Baroudi et al. [2014] for an isotropic circular cylinder. It is seen from Figs. 5-7 that the anisotropy and viscous coupling do not affect the modal shapes.

\section{Conclusion}

An exact method has been presented for studying the coupled structural-fluid analysis of a three-layer transversely isotropic finite circular cylinder filled with a viscous fluid using the wave propagation approach. For uncoupled analysis in the case of one-layer model, the natural frequencies of the transversely isotropic cylinder by the present method are compared with the results available in Kudlicka [2006]. For coupled analysis the comparisons of the frequencies by the present method and numerical FEM are carried out. Through the comparisons it is possible to conclude that the present method is correct. It is concluded that the methodology proposed by this paper can be widely applied to infinite number of layers. This study takes into consideration the effect of fluid viscosity and the effect of layers, both of these factors are important in engineering. This method can be 
easily applied to the measurement of the fluid viscosity in the case of submerged structures.

\section{Acknowledgment}

We would like to sincerely thank Prof. A. Ambari for his useful comments and suggestions.

\section{Appendix}

Elements of the matrix given in Eq. (16) is as follows:

$$
\begin{aligned}
& M_{11}=\eta\left[a J_{1}^{\prime}(\delta a)-J_{1}(\delta a)\right] \\
& M_{12}=C_{66}^{(1)}\left[a J_{1}^{\prime}\left(\beta_{1} a\right)-J_{1}\left(\beta_{1} a\right)\right] \\
& M_{13}=C_{66}^{(1)}\left[a Y_{1}^{\prime}\left(\beta_{1} a\right)-Y_{1}\left(\beta_{1} a\right)\right] \\
& M_{21}=J_{1}(\delta a) \\
& M_{22}=\nu \alpha^{2} J_{1}\left(\beta_{1} a\right) \\
& M_{23}=\nu \alpha^{2} Y_{1}\left(\beta_{1} a\right) \\
& M_{32}=J_{1}\left(\beta_{1} a_{1}\right) \\
& M_{33}=Y_{1}\left(\beta_{1} a_{1}\right) \\
& M_{34}=J_{1}\left(\beta_{2} a_{1}\right) \\
& M_{35}=Y_{1}\left(\beta_{2} a_{1}\right) \\
& M_{42}=C_{66}^{(1)}\left[a_{1} J_{1}^{\prime}\left(\beta_{1} a_{1}\right)-J_{1}\left(\beta_{1} a_{1}\right)\right] \\
& M_{43}=C_{66}^{(1)}\left[a_{1} Y_{1}^{\prime}\left(\beta_{1} a_{1}\right)-Y_{1}\left(\beta_{1} a_{1}\right)\right] \\
& M_{44}=\mu_{2}\left[a_{1} J_{1}^{\prime}\left(\beta_{2} a_{1}\right)-J_{1}\left(\beta_{2} a_{1}\right)\right] \\
& M_{45}=\mu_{2}\left[a_{1} Y_{1}^{\prime}\left(\beta_{2} a_{1}\right)-Y_{1}\left(\beta_{2} a_{1}\right)\right] \\
& M_{54}=J_{1}\left(\beta_{2} a_{2}\right) \\
& M_{55}=Y_{1}\left(\beta_{2} a_{2}\right) \\
& M_{56}=J_{1}\left(\beta_{3} a_{2}\right) \\
& M_{57}=Y_{1}\left(\beta_{3} a_{2}\right) \\
& M_{64}=\mu_{2}\left[a_{2} J_{1}^{\prime}\left(\beta_{2} a_{2}\right)-J_{1}\left(\beta_{2} a_{2}\right)\right] \\
& M_{65}=\mu_{2}\left[a_{2} Y_{1}^{\prime}\left(\beta_{2} a_{2}\right)-Y_{1}\left(\beta_{2} a_{2}\right)\right] \\
& M_{66}=C_{66}^{(3)}\left[a_{2} J_{1}^{\prime}\left(\beta_{3} a_{2}\right)-J_{1}\left(\beta_{3} a_{2}\right)\right] \\
& M_{67}=C_{66}^{(3)}\left[a_{2} Y_{1}^{\prime}\left(\beta_{3} a_{2}\right)-Y_{1}\left(\beta_{3} a_{2}\right)\right] \\
& \left.M_{1}\right)
\end{aligned}
$$




$$
\begin{aligned}
& M_{76}=a_{3} J_{1}^{\prime}\left(\beta_{3} a_{3}\right)-J_{1}\left(\beta_{3} a_{3}\right) \\
& M_{77}=a_{3} Y_{1}^{\prime}\left(\beta_{3} a_{3}\right)-Y_{1}\left(\beta_{3} a_{3}\right)
\end{aligned}
$$

\section{References}

Armenakas, A. E. [1965] "Torsional waves in composite rods," Journal of the Acoustical Society of America 38, 439-446.

Carcione, J. M. and Seriani, G. [1994] "Torsional oscillations of anisotropic hollow circular cylinders," Acoustics Letters 18(6).

Comsol Multiphysics, Analysis User's Manual Version 3.5a, November 2008.

El Baroudi, A., Razafimahery, F. and Rakotomanana, L. [2014] "Fluid structure interaction within three-dimensional models of an idealized arterial wall," International Journal of Engineering Science 84, 113-126.

Gazis, D. C. [1959a] "Three-dimensional investigation of the propagation of waves in hollow circular cylinders, I : Analytical foundation," Journal of the Acoustical Society of America 31(5), 568-573.

Gazis, D. C. [1959b] "Three-dimensional investigation of the propagation of waves in hollow circular cylinders, II : Numerical results," Journal of the Acoustical Society of America 31(5), 573-578.

Kaul, R. K. and Shaw, R. P. [1981] "Torsional waves in an axially homogeneous bimetallic cylinder," International Journal of Solids and Structures 17, 379-394.

Kim, J. O. and Chun, H. Y. [2003] "Interaction between the torsional vibration of a circular rod and an adjacent viscous fluid," Journal of Vibration and Acoustics 125, 39-45.

Kim, J. O., Wang, Y. and Bau, H. H. [1991] "The effect of an adjacent viscous fluid on the transmission of torsional stress waves in a submerged waveguide," Journal of the Acoustical Society of America 89(3), 1414-1422.

Kudlicka, J. [2006] "Dispersion of torsional waves in thick-walled transversely isotropic circular cylinder of infinite length," Journal of Sound and Vibration 294, 368-373.

Markus, S. and Mead, D. J. [1996] "Wave motion in a three-layered, orthotropic-isotropicorthotropic, composite shell," Journal of Sound and Vibration 181(1), 149-167.

Mirsky, I. [1965] "Wave propagation in transversely isotropic circular cylinders Part I: Theory," Journal of the Acoustical Society of America 37(6), 1016-1021.

Misra, J. C. [1983] "Propagation of torsional waves in tubular bones," Journal of Mathematical Analysis and Applications 96, 313-329.

Morse, M and Feshbach, H. R. [1946] Methods of Theoretical Physics (McGraw-Hill, New York).

Wolfram Research Mathematica Version 10.1, Champaign, Illinois 2015. 